\title{
Stability of Difference Approximations of Dissipative Type for Mixed Initial-Boundary Value Problems. I*
}

\author{
By Stanley Osher
}

Abstract. H. O. Kreiss, [2], has recently extended the stability theory of difference approximations to include the hyperbolic system

$$
u_{t}=A u_{x}, \quad 0 \leqq x, t,
$$

with $A$ a diagonal matrix. Appropriate boundary and initial conditions are given. The amplification matrix $\hat{Q}(\xi)$ need not be diagonal. However, he required that $|\hat{Q}(\xi)| \leqq 1$. We use certain results in matrix theory and Wiener-Hopf factorization to replace this restrictive assumption by certain reasonable assumptions on accuracy of $\hat{Q}(\xi)$ and smoothness of an associated positive-definite symmetric matrix. This technique will be important in half-space problems in many space variables since for such problems the amplification matrix will certainly not be diagonal.

1. Introduction. The purpose of this paper is to replace the restrictive Assumption 4 of Kreiss [2] by assumptions on the accuracy of the difference approximation and the smoothness of an associated positive-definite symmetric matrix. We shall use the notation in [2] throughout. Equation numbers surrounded by parentheses will refer to [2], those with only a right-hand parenthesis refer to this paper.

This technique may be used for multidimensional problems if we assume appropriate analogous hypotheses.

The basic assumption and theorems which we shall use are the following:

I. Theorem (Osher [1]). Let $L$ be a bounded linear operator on a Hilbert space $H$. Let $P$ be an orthogonal projection on $H$. Assume

1.1) $\|(I-P) L\| \leqq 1$.

1.2) $\|L(I-P)\| \leqq 1$.

1.3) There exists a sequence of nonnegative numbers $\left\{a_{n}\right\}$ such that $\sum_{n=0}^{\infty} a_{n}=a<\infty$ and $\left\|P L^{n} P\right\| \leqq a_{n}$ for $n=0,1,2, \cdots$.

Then

$$
\left\|L^{n}\right\| \leqq\left(1+a^{2}\right)^{1 / 2} \text { for all } n=0,1,2, \cdots .
$$

II. Theorem (Kreiss [5]). Let (1.6) have order of accuracy $2 s-1$ with respect to (1.1). Let assumptions (1), (2), and (3) of [2] be valid. Then there exists a positivedefinite Hermitian matrix $\mathfrak{F}\left(e^{i \xi}\right)$ such that

$$
c^{-1} I \leqq \mathcal{C}\left(e^{i \xi}\right) \leqq c I
$$

for some constant $c>0$ independent of $\xi$. Furthermore, the matrix

Received April 18, 1968, revised July 16, 1968.

* Research carried out at Brookhaven National Laboratory under contract with the U. S. Atomic Energy Commission. 


$$
A\left(e^{i \xi}\right)=\sum_{j=-r}^{p} A_{j} e^{-i j \xi}
$$

satisfies

$$
A^{*}\left(e^{i \xi}\right) \mathcal{H C}\left(e^{i \xi}\right) A\left(e^{i \xi}\right) \leqq \mathfrak{C}\left(e^{i \xi}\right) .
$$

III. Assumption. Let $\mathfrak{K}\left(e^{i \xi}\right)$ have a finite Fourier expansion

$$
\mathfrak{F}\left(e^{i \xi}\right)=\sum_{j=-t}^{t} \mathfrak{F}_{j} e^{i \xi j} .
$$

(We think that such an $\mathcal{H C}$ can be obtained if the $A_{j}$ are symmetric. However, we have not yet proven this, and in fact we expect that there are probably more general conditions which may be used.)

IV. Theorem (Gohberg and KreIn [4]). Let $\mathfrak{H C}\left(e^{i \xi}\right)$ have the above properties. Then we may write

$$
\mathscr{H}\left(e^{i \xi}\right)=B^{*}\left(e^{i \xi}\right) B\left(e^{i \xi}\right) \quad\left(B^{*} \text { is the conjugate transpose of } B\right)
$$

where

$$
B\left(e^{i \xi}\right)=\sum_{j=0}^{t} B_{j} e^{i j \xi}
$$

and

$$
\operatorname{det}[B(x)] \neq 0 \text { for }|x| \leqq 1 .
$$

We may now state our Main Theorem.

Main Theorem. The approximation to the initial-boundary value problem is stable if:

1.12) The assumptions (1), (2), (3), are fulfilled and (4) is replaced by letting 1.6) have order of accuracy $2 s-1$ with respect to 1.1$)$ and requiring that $\mathcal{H}\left(e^{i \xi}\right)$ satisfy Assumption III. (We may sometimes make a change of variables so that the accuracy assumption becomes valid, see [5].)

1.13) $z_{0}=1$ is not a generalized eigenvalue of $q$.

1.14) $q$ has no eigenvalues $z_{0}$, with $\left|z_{0}\right|>1, z_{0} \neq 1$.

2. Renorming the Hilbert Space. Kreiss uses the Hilbert space $H$, which is the space of all grid-functions $\omega_{\nu}$ defined for $\nu>-r$ which fulfill the boundary conditions and for which

$$
\|\omega\|^{2}=\sum_{\nu=1}^{\infty}\left|\omega_{\nu}\right|^{2} h<\infty .
$$

The Fourier transform space $\hat{H}$ of functions of the form

$$
\hat{\omega}\left(e^{i \xi}\right)=\sum_{\nu=1}^{\infty} \omega_{\nu} e^{i \nu \xi}
$$

is isometrically isomorphic to $H$.

Let us consider the Hilbert space $H_{(-\infty, \infty)}$ of grid-functions $\omega_{\nu}$ defined for $-\infty<\nu<\infty$ which fulfill the boundary conditions and for which 


$$
\sum_{\nu=-\infty}^{\infty}\left|\omega_{\nu}\right|^{2} h<\infty
$$

We also consider its Fourier transform space $\hat{H}_{(-\infty, \infty)}$.

On $\hat{H}_{(-\infty, \infty)}$, we define the orthogonal projection

$$
P_{+}\left(\sum_{\nu=-\infty}^{\infty} \omega_{\nu} e^{i \nu \xi}\right)=\sum_{\nu=-r+1}^{\infty} \omega_{\nu} e^{i \nu \xi}
$$

Let

$$
P_{+} \hat{H}_{(-\infty, \infty)}=\hat{G}
$$

define the space $\hat{G}$, and consider its inverse Fourier transform space $G$. However, on $\hat{G}$, we shall use a new equivalent norm which we define as

$$
\|\hat{\omega}\|_{B}=(B \hat{\omega}, B \hat{\omega})^{1 / 2}
$$

where $B\left(e^{i \xi}\right)$ is defined in Theorem II, and

$$
(\hat{f}, \hat{g})=\frac{1}{2 \pi} \sum_{k=1}^{n} \int \hat{f}\left(k, e^{i \xi}\right) \overline{\hat{g}\left(k, ! e^{i \xi}\right)} d \xi
$$

where $\bar{a}$ denotes the complex conjugate of $a$. From [4], we know that

$$
\hat{u}=B \hat{\omega}
$$

defines a bounded, linear, invertible operator on $\hat{G}$. Hence, it follows that $\exists k_{B}{ }^{\prime}$, $k_{B}{ }^{\prime \prime}>0$ with

$$
k_{B}{ }^{\prime}(\hat{\omega}, \hat{\omega})^{1 / 2} \leqq\|\hat{\omega}\|_{B} \leqq k_{B}{ }^{\prime \prime}(\hat{\omega}, \hat{\omega})^{1 / 2} .
$$

Thus, the renormed space $\hat{G}_{B}$ is equivalent to $\hat{G}$. We shall obtain stability on the space $\hat{G}_{B}$.

3. The Operators $T$ and $S$. The initial-boundary approximation differs from

$$
w_{j}(t+k)=\sum_{\nu=\max (-r, 1-j-r)}^{p} A_{\nu} E^{\nu} w_{j}(t), \quad j=-r+1,-r+2, \cdots,
$$

where $E w_{j}=w_{j+1}$, by a boundary perturbation

$$
\begin{aligned}
u_{j}(t+k)= & -\sum_{\nu=1-j-r}^{p} A_{\nu} E^{\nu} u_{j}(t) \\
& +\sum_{p=1}^{s} C_{p j} u_{p}(t+k), \text { for } j=-r+1,-r+2, \cdots, 0 ;
\end{aligned}
$$

and

$$
u_{j}(t+k)=0, \quad j=1,2, \cdots .
$$

Equation 3.1) defines a Toeplitz operator $T$ on $G_{B}$ (and $\hat{T}$ on $\hat{G}_{B}$ ). Equation (3.2) defines a finite-dimensional operator $S$ (and $\hat{S}$ ). We have thus shown that proving stability of the initial-boundary approximation is equivalent to bounding

$$
\left\|(\hat{T}+\hat{S})^{n}\right\|_{B} \leqq k, \quad n=0,1,2, \cdots
$$

for $k$ independent of $n$. 
4. Orthogonal Projections on $\hat{G}_{B}$. We claim that the set of vectors

4.1) $\quad\left\{B^{-1}\left(e^{i \xi}\right) f_{\nu} e^{i j \xi}\right\}=\hat{g}_{\nu j}\left(e^{i \xi}\right), \quad \nu=1,2, \cdots, n, \quad j=-r+1,-r+2, \cdots$, where $f_{\nu}$ is the $\nu$ th unit $n$ vector, form a complete orthonormal set on $\hat{G}_{B}$. Suppose for all $\nu, j$

$$
\left(\hat{v}, \hat{g}_{v j}\right)_{B}=0 \Leftrightarrow\left(B \hat{v}, f_{\nu} e^{i j \xi}\right)=0 \Leftrightarrow B \hat{v}=0 \Rightarrow \hat{v}=0 .
$$

This proves completeness. Orthonormality also follows simply. Thus, any element on $\hat{G}_{B}$ may be written as

$$
\hat{v}\left(e^{i \xi}\right)=\sum_{\nu=1}^{n} \sum_{j=-r+1}^{\infty}\left(B \hat{v}, f_{\nu} e^{i j \xi}\right) \hat{g}_{\nu j}\left(e^{i \xi}\right) .
$$

We define the orthogonal projector on $\hat{G}_{B}$

$$
(I-P) \hat{v}=\sum_{\nu=1}^{n} \sum_{j=N+1}^{\infty}\left(B \hat{v}, f_{\nu} e^{i j \xi}\right) \hat{g}_{v j}\left(e^{i \xi}\right),
$$

where $N>0$ is defined below.

The following lemma is basic to this work.

IV. Lemma. Let $N=\max (t, s+p, p-r)$. Then

$$
\begin{aligned}
& \|(I-P)(\hat{T}+\hat{S})\|_{B} \leqq 1, \\
& \|(\hat{T}+\hat{S})(I-P)\|_{B} \leqq 1 .
\end{aligned}
$$

Proof. We shall show that the difference operator $\hat{T}+\hat{S}$ acting on the space $(I-P) \hat{G}_{B}$ and the restriction $(I-P)(\hat{T}+\hat{S}) \hat{G}_{B}$ both behave like the associated pure initial-value problems $A\left(e^{i \xi}\right)(I-P) \hat{G}_{B}$ and $(I-P) A\left(e^{i \xi}\right) \hat{G}_{B}$. We then use 1.7) to prove the Lemma.

More precisely, we claim for any $\hat{w}\left(e^{i \xi}\right)$ in $\hat{G}_{B}$ that

4.5) $(I-P) \hat{S}=\hat{S}(I-P)=0$,

4.6) $(I-P) \hat{T} \hat{w}=(I-P) A\left(e^{i \xi}\right) \hat{w}\left(e^{i \xi}\right)$, and

4.7) $\hat{T}(I-P) \hat{w}=A\left(e^{i \xi}\right)(I-P) \hat{w}\left(e^{i \xi}\right)$.

We know that $B\left(e^{i \xi}\right) \hat{S} \hat{w}\left(e^{i \xi}\right)$ has a finite Fourier expansion because of 1.10):

$$
B\left(e^{i \xi}\right) \hat{S} \hat{w}\left(e^{i \xi}\right)=\sum_{j=-r+1}^{t} v_{j} e^{i j \xi} .
$$

Furthermore, $\hat{S}$ annihilates all vectors

$$
\hat{v}\left(e^{i \xi}\right)=\sum_{j=s+p+1}^{\infty} v_{j} e^{i j \xi}
$$

The range and support of $\hat{S}$ both lie in the support of $P$, i.e., near the boundary, and thus 4.5) is valid.

Next we notice that

$$
A\left(e^{i \xi}\right)(I-P) \hat{w}\left(e^{i \xi}\right)
$$

is the same as

$$
\hat{T}(I-P) \hat{w}\left(e^{i \xi}\right)
$$

because the range of $A\left(e^{i \xi}\right)(I-P)$ is contained in $\hat{G}_{B}$. Thus 


$$
\begin{aligned}
\|\hat{T}(I-P) \hat{w}\|_{B} & \left.=\left\|A\left(e^{i \xi}\right)(I-P) \hat{w}\right\|_{B} \leqq\|(I-P) \hat{w}\|_{B} \quad(\text { by } 1.7)\right) \\
& \leqq\|\hat{w}\|_{B} .
\end{aligned}
$$

Finally, we see that the $j$ th Fourier coefficient of

$$
B P_{+} A\left(e^{i \xi}\right) \hat{v}\left(e^{i \xi}\right)
$$

and

$$
B A\left(e^{i \xi}\right) \hat{v}\left(e^{i \xi}\right)
$$

are equal for $j \geqq t-r+1$ because of 1.10). Thus,

$$
\begin{aligned}
\|(I-P) \hat{T} \hat{v}\|_{B} & =\left\|(I-P) A\left(e^{i \xi}\right) \hat{v}\left(e^{i \xi}\right)\right\|_{B} \\
& \left.\leqq\left\|A\left(e^{i \xi}\right) \hat{v}\left(e^{i \xi}\right)\right\|_{B} \leqq\|\hat{v}\|_{B} \quad(\text { by 1.7 })\right) .
\end{aligned}
$$

This, along with 4.11), yields

$$
\begin{aligned}
& \|(I-P) \hat{T}\|_{B} \leqq 1, \\
& \|\hat{T}(I-P)\|_{B} \leqq 1 . \quad \text { Q.E.D. }
\end{aligned}
$$

Hence, in order to prove stability, we need only verify condition (3) of Theorem I for $\left\|P(\hat{T}+\hat{S})^{n} P\right\|_{B}$.

5. Proof of Main Theorem. Kreiss has shown in [2] that if the hypotheses of our Main Theorem are valid, then $(T+S-z)^{-1}$ exists for $|z| \geqq 1, z \neq 1$. Moreover, let

$$
\hat{v}\left(e^{i \xi}\right)=(\hat{T}+\hat{S}-z I)^{-1} \hat{w}\left(e^{i \xi}\right)
$$

where $v_{j}=\sum_{\nu=-r+1}^{\infty} k_{j \nu}(z) w_{\nu}$. He has also shown that each $k_{j \nu}(z)$ is analytic in some region $|z| \geqq 1-\rho, \rho>0$, and that for any $\delta>0$, there is an $\epsilon>0$, with $\epsilon<\rho$ such that

$$
\left\|\left|k_{j \nu}(z)\right|\right\| \leqq c^{\prime}(\epsilon)(1+\delta)^{|\nu-j|} \text { for all } z \text { with }|z| \geqq 1-\epsilon,
$$

and $c^{\prime}>0$ independent of $\delta, j, \nu$ and $z$. \|\|$\|$ denotes the norm of an $n \times n$ matrix.

We shall use these results to show that $P(T+S-z I)^{-1} P$ is a uniformly bounded, analytic operator for $|z| \geqq 1-\epsilon$. It then follows that

$$
\left\|P(\hat{T}+\hat{S})^{n} P\right\|_{B} \leqq c^{\prime \prime}(1-\epsilon / 2)^{n}
$$

and we have stability. We shall consider

$$
\begin{aligned}
& B P(\hat{T}+\hat{S}-z I)^{-1} P B^{-1} \hat{v} \\
& =\sum_{p=-r+1}^{N} \sum_{k=1}^{n} f_{k} e^{i p \xi}\left(B[\hat{T}+\hat{S}-z I]^{-1} B^{-1} \sum_{\nu=1}^{n} \sum_{j=-r+1}^{N}\left(\hat{v}, f_{\nu} e^{i j \xi}\right) f_{\nu} e^{i j \xi}, f_{k} e^{i p \xi}\right) .
\end{aligned}
$$

Hence, for analyticity, it is sufficient that

$$
\sum_{k=1}^{n} \sum_{p=-r+1}^{N}\left(B[\hat{T}+\hat{S}-z]^{-1} B^{-1} \sum_{\nu=1}^{n} \sum_{p=-r+1}^{N} f_{\nu} e^{i j \xi}, f_{k} e^{i p \xi}\right)
$$

be analytic.

Define the matrices $B_{(k)}^{(-1)}$ by 


$$
B^{-1}\left(e^{i \xi}\right)=\sum_{k=0}^{\infty} B_{(k)}^{(-1)} e^{i k \xi} .
$$

From the properties of $B$, and the Cauchy integral formula, it is clear that there is an estimate

$$
\left\|\left|B_{(k)}^{(-1)}\right|\right\| \leqq c^{\prime \prime \prime}\left(1-\delta_{0}\right)^{k} \text { for } \delta_{0}>0, c^{\prime \prime \prime}>0, \text { and all } k .
$$

We must show that

$$
\sum_{\nu=\nu_{0}}^{\infty} k_{j \nu}(z) B_{\left(\nu-\nu_{0}\right)}^{(-1)} \quad \text { for }-r+1 \leqq \nu_{0} \leqq N,-r+1 \leqq j \leqq N
$$

converges uniformly for $|z|>1-\epsilon>1-\rho, \epsilon>0$.

In 5.2), take $\delta=\delta_{0}$ of 5.7). Then

$$
\begin{aligned}
\sum_{\nu=\nu_{0}}^{\infty}\left\|k_{j \nu}(z) B_{\left(\nu-\nu_{0}\right)}\right\| & \leqq c^{\prime}(\epsilon) \sum_{\nu=\nu_{0}}^{\infty}\left(1+\delta_{0}\right)^{|\nu-j|} c^{\prime \prime \prime}\left(1-\delta_{0}\right)^{\nu-\nu_{0}} \\
& \leqq c^{\mathrm{iv}} / \delta_{0}{ }^{2} \text { if }|z| \geqq 1-\epsilon .
\end{aligned}
$$

Thus the sum is the uniform limit of analytic matrices and is hence analytic. Q.E.D.

Department of Mathematics

University of California

Berkeley, California 94720

1. S. OsHER, "Systems of difference equations with general homogeneous boundary conditions," Trans. Amer. Math. Soc., v. 137, 1969, pp. 177-201.

2. H. O. KREISs, "Stability theory for difference approximations for mixed initial boundary value problems. I," Math. Comp., v. 22, 1968, pp. 703-714.

3. H. O. KREISS, "Difference approximations for the initial-boundary value problem for hyperbolic differential equations," in Numerical Solutions of Nonlinear Differential Equations (Proc. Adv. Sympos., Madison, Wis., 1966), Wiley, New York, 1966, pp. 141-166. MR 35 \#5156.

4. I. C. GOHBERG \& M. G. KREIN, "Systems of integral equations on a half line with kernels depending on the difference of arguments," Uspehi Mat. Nauk, v. 13, 1958, no. 2 (80), pp. 3-72; English transl., Amer. Math. Soc. Transl., v. (2) 14, 1960, pp. 217-288. MR 21 \#1506; MR 22 $\# 3954$.

5. H. O. KREISs, "On difference approximations of the dissipative type for hyperbolic differential equations," Comm. Pure Appl. Math., v. 17, 1964, pp. 335-353. MR 29 \#4210. 